\title{
Reminiscences and lessons on the occasion of a silver jubilee
}

\author{
C. Th. J. Alkemade \\ Fysisch Laboratorium der Rijksuniversiteit Utrecht, Princetonplein 5, 3584 CC Utrecht, The Netherlands
}

(Received 28 July 1980)

\begin{abstract}
This paper, written to commemorate the publication of WALSH's classical paper on the potentialities of atomic absorption spectroscopy (AAS) 25 years ago, contains nothing new. It is a personal recollection of the work done independently at our laboratory prior to 1955 which resulted in the construction of a "negative filter" (for use in atomic emission spectroscopy) and an "absorption flame photometer." Some lessons are drawn from the historical development of AAS about the inventing of new analytical methods in general.
\end{abstract}

\section{REMinisCENCES}

ON A HOT summer day in 1955, when doing low-temperature work at the laboratory of the National Research Council at Ottawa, I was surprised to receive a letter from Australia, which had been sent on to me via my home laboratory. In this letter I was asked if I had applied for a patent (or intended to do so) on an analytical method described as "absorption flame photometry" in a paper [1] and in a Letter to the Editor [2] by J. M. W. MiLATZ and myself. It turned out that at two, nearly opposite sites on the globe (as well as in the alphabet) we and a certain A. WALSH, independently and simultaneously, had had the same brainwave. This brainwave had "materialized" in two papers [1] and [3], respectively, whose dates of receipt differed by no more than the time it takes for a manuscript to reach Europe from Australia. The looks of the two papers however were quite different. WALsh's paper was a thorough, systematic and theoretical exposition of the potentialities of atomic absorption analysis. Ours on the other hand was purely experimental* and described an absorption flame photometer as well as a "negative-filter" instrument (see below).

Of course, I was flattered to think that at least someone had taken notice of our paper, which sadly appeared in a journal completely unknown to analytical chemists. I was also surprised to discover that a piece of work, which I had omitted from my thesis [4] as being not particularly urgent, was considered by others to be worth patenting. In 1954 I thought it was not worth paying an extra hundred guilders out of my pocket to include a chapter on this topic in my thesis. I contented myself instead with a brief formulation of the idea in one of the Dutch "Stellingen" (Assertions) which have to accompany every thesis submitted in our country. As a matter of fact, I had actually applied atomic absorption in my thesis work, but only as an expedient for monitoring atomic sodium concentrations in a flame. $\ddagger$

Since as a post-doctoral fellow at the N.R.C. I had descended from high-temperature flames to low-temperature solid-state physics, I had (temporarily) lost interest in flames and so I was quick to reply that we had no intentions whatsoever of applying for a

\footnotetext{
* This paper, and the present one I am afraid, are the only ones I have ever written without using any mathematical equation.

$\dagger$ Another Assertion referred to the use of wavelength-modulation for discriminating a weak, narrow emission or absorption line from a continuous background spectrum.

$\ddagger$ For a more detailed historical account of preliminary reports on analytical atomic absorption spectroscopy see Ref. [5].

[1] C. Th. J. Alkemade and J. M. W. Milatz, Appl. Sci. Res. B4, 289 (1955).

[2] C. Th. J. Alkemade and J. M. W. Milatz, J. Opt. Soc. Am. 45, 583 (1955).

[3] A. Walsh, Spectrochim. Acta 7, 108 (1955).

[4] C. TH. J. AlkemaDE, A contribution to the understanding and development of flame photometry, Ph.D. Thesis, Utrecht (1954).

[5] R. Mavrodineanu and H. Borteux, Flame Spectroscopy. Wiley, New York (1965).
} 
patent. The pleasure that my former students R. Rikmenspoel and E. W. DE VRIES and I have experienced in playing around with this new gadget had been a reward in itself.

The roots of atomic absorption in the past century, its early application in physics and in astrophysics (Fraunhofer lines) and its occasional analytical application to detect mercury vapour have been described frequently. But none of this explains why at two different places atomic absorption spectroscopy as an all-round analytical method was triggered 25 years ago.

In Utrecht the idea was put forward more or less accidentally. Although its history is not particularly interesting, I shall summarize it and draw some general historical lessons from it.

My promotor, Professor J. M. W. MIlatz, then director of the Fysisch Laboratorium of our University, had occasionally challenged me to develop-as an interlude in my thesis work-a negative-filter technique for $\mathrm{Li}$-isotope analysis by atomic emission spectroscopy. The isotopic shift of the first resonance doublet of this atom is very small and difficult to resolve by conventional spectral selectors. Furthermore, even if sufficient spectral resolution was available, it would be difficult to prevent the wavelength-setting of the spectral selector from drifting away.

In our laboratory a dispersion-less i.r. molecular absorption spectrometer had been developed not long before for $\mathrm{CO}_{2}$-isotope analysis [6]. The selective detector consisted of a closed cell which was filled with isotopically pure $\mathrm{CO}_{2}$ gas and connected to a condenser microphone which measured the pressure rise due to absorption of radiation. In this way the detector responded only to wavelengths that are characteristic for the isotope contained in the detector cell. An "optical bridge" with mechanical intensity-modulation and a.c. detection was used to obtain zero-balance when the $\mathrm{CO}_{2}$-isotope composition in the sample cell was the same as in a reference cell. Because the selectivity of the detector was based on the absorption properties of the gas in the detector cell (and not on the transmission properties as with a normal filter), the term negative filter was devised.

Following the same basic idea I built the experimental set-up schematically shown in Fig. 1 (reproduced from our original publication). The sample containing the unknown Li-isotope mixture was supposed to be introduced, atomized and excited in an appropriate light source S. Two light beams I and II were formed and alternately chopped by a rotating disk $C$ with openings. In one of the beams (II) a reference absorption cell $\mathrm{A}$ had to be inserted containing a pure $\mathrm{Li}$ isotope at a fixed vapour pressure. The two beams were detected by the same photomultiplier tube and a synchronous a.c. meter was used to read the off-balance of the optical a.c. bridge. The intention was to obtain in this way a reading that was a measure for the intensity of the line emitted in $\mathrm{S}$ by the same isotopic species as contained in $\mathrm{A}$.

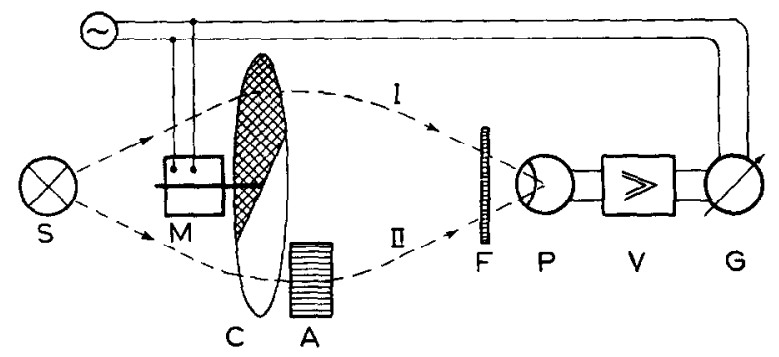

Fig. 1. Scheme of experimental arrangement. I and II are two beams of light emitted by source $\mathrm{S} ; \mathrm{C}=$ periodic chopper that modulates the two light-beams in opposite phase; $\mathrm{M}=$ motor fed by a.c. line voltage and driving chopper disk $\mathrm{C} ; \mathrm{A}=$ medium with selective spectral absorbing power; $F=$ conventional spectral broad-band filter; $P=$ photomultiplier tube; $V=$ electronic a.c. amplifier; $G=$ a.c. galvanometer acting as a synchronous (phase- and frequency-sensitive) meter because its electromagnet is fed by a.c. line voltage (reproduced from Ref. [1]). 
I must confess that I failed to achieve this aim because of the difficulties I encountered in producing a stable $\mathrm{Li}$ vapour in a heated cell. In fact I did not try very hard, as I was already acquainted with flames and found them ideally suited for producing an absorbing alkali vapour at a constant and easily controllable concentration. The thermal emission of the absorbing flame placed at position $\mathrm{A}$ in Fig. $1 \mathrm{did}$ not interfere because a.c. detection was applied. However, the Doppler and collisional broadening of the spectral lines in the absorbing flame spoiled the spectral selectivity needed for Li-isotope analysis. But the same arrangement could be used to select, for example, the $\mathrm{Na}-\mathrm{D}$ resonance lines emitted by $\mathrm{S}$ (for example, an analytical flame) with a still appreciable resolution (better than $100 \mathrm{~m} \hat{\Lambda}$ ). This negative-filter technique was by its very nature element-specific and its wavelength-setting could not drift away. Moreover, its optical conductance (luminosity) was not limited by narrow slits as in a monochromator. In principle no extra, conventional spectral selectors were needed, but in practice we inserted a broad-band interference filter (bandwidth $\approx 100 \AA \AA$ ) to eliminate the wavelength-dependence of the zero-balance. This dependence was caused by the unequal chromatic aberrations of the lenses used in the two beams. The use of mirrors would have eliminated this problem, of course. But the insertion of a filter was also useful in reducing the noises due to the background emissions of the absorbing flame in $\mathrm{A}$ and of the analytical excitation source in $\mathrm{S}$.

It was now but a small step to convert this negative-filter instrument (for application in emission spectroscopy) into a flame atomic absorption spectrometer. I only had to place the analytical flame in position $\mathrm{A}$ while choosing for $\mathrm{S}$ a constant atomic line source, e.g. another flame or a metal-vapour lamp, emitting a resonance line of the element to be determined. In this way the absorption by the analyte atoms in flame A was sclectively measured and converted as usual into sample concentration by means of an analytical curve. To cite Ref. [1] (p. 292):

"The application of the latter variant might (!) be considered as an example of a new branch of flame photometry: the absorption flame photometry."

It did not require much ingenuity on my part to make this invention, and I did not consider it a great achievement.

An inherent advantage of this particular double-beam arrangement was that the base-line (100\% transmission) was not affected by drift in the intensity of the atomic line source $\mathrm{S}$.

We tested the performance of the set-up as a negative filter by using it to demonstrate clearly the occurrence of self-reversal in the $\mathrm{Na}$ emission of an unshielded flame at high concentrations. When used in the AAS mode, with a Na street lamp placed at $S$, the useful dynamic range appeared to be $0.1-30 \mathrm{mg} / 1$ for $\mathrm{Na}$ solutions, while the detection limit was $0.03 \mathrm{mg} / \mathrm{l} \mathrm{Na}$ (which corresponded to about $0.1 \%$ absorption). Although the set-up was not optimized for use in AAS, the latter value compared well with values reported for Na by Russell, SHELton and WALSH [7] and obtained with the first commercial single-beam instruments $[5,8]$.

The basic idea of the negative-filter technique was later taken up again in atomic spectroscopy by BOWMAN, SULLIVAN and WALSH [9] who applied a pulsating metal vapour cloud in a sputtering chamber for selective modulation of resonance lines.

Recently, HIEFTJE and collaborators $[10,11]$ have applied this alternative negativefilter technique in atomic absorption measurements with a continuum background

[7] B. J. Russell, J. P. Shelton and A. Walsh, Spectrochim. Acta 8, 317 (1957).

[8] A. C. Menzies, Anal. Chem. 32, 898 (1960).

[9] J. A. Bowman, J. V. Sullivan and A. Walsh, Spectrochim. Acta 22, 205 (1966).

[10] R. L. Cohran and G. M. HieftJe, Anal. Chem. 49, 98 (1977); 50, 791 (1978).

[11] J. Shabushnig, S. Downey and G. M. HieftJe, 6th Annual Meeting FACSS, 16-21 September (1979), Philadelphia (Pa.), Book of Abstracts, paper No. 190. 
source. HANNAFORD demonstrated the sharpening of atomic resonance lines resulting from this selective-modulation technique [12]. HANNAFORD and HoRN [13] also noted the close analogy between our negative-filter technique and their technique for isolating recoil-less $\gamma$-rays in Mössbauer spectroscopy.

An auxiliary flame was also used by RANN [14] as an atomic line source in his investigations into the feasibility of absolute analysis by AAS.

In 1963 KAHN and SLAVIN [15] described a commercial double-beam flame atomic absorption spectrometer that was based essentially on the same scheme as that shown in Fig. 1. They also used mechanical chopping of the two beams at opposite phases, in combination with a single photodetector and synchronous detection. Their use of mirrors instead of lenses in the optical system was a major improvement.

Several authors, who later erroneously argued that FAAS is essentially more sensitive than the flame atomic emission method because most atoms are in their ground-state, had unfortunately overlooked a pertinent statement on p. 292 in our original paper [1]. We stated there that the gain in sensitivity was due to the fact that the effective excitation temperature of the discharge line source was higher than the temperature of the absorbing flame. If both temperatures were equal, there would be no gain in sensitivity (for a further discussion see also Ref. [16]).

\section{LESSONS}

(i) Inventions do not drop from the sky, neither are they produced as the result of a logical, straightforward reasoning process. Frequently they need an incubation period. In our case this period was characterized by small, rather accidental steps in the development of the set-up, which was accompanied by trial and error. As it occurs more often, the final result was different from the original intention and a surprise factor was included. In such situations it is important that one keeps an open mind and is constantly on the outlook for alternative solutions or applications. In retrospect, one wonders if in the future this surprise factor could not be reduced by exploring new possible analytical methods more systematically. An excellent example of such an exploration has been given by WALSH in his paper [17]: "Atomic spectroscopy-what next?" In this paper he presents a number of feasible experimental arrangements involving atomic spectral lamps, conventional as well as separated flames, sputtering chambers, etc. in various orders and with various functions. He manipulates atomic absorption and atomic fluorescence just as an organist pulls out different stops. In such a way one can weigh up the advantages and disadvantages of a number of new methods at the same time and "choose" the invention that fits best to one's purposes.

(ii) History does not proceed abruptly; there is evolution rather than revolution; the same is true in applied science. New analytical methods usually have many links with preceding, well-established methods. There was much more continuity in the changeover from the flame emission to the flame absorption method than was often realized during the first tumultuous explosion of AAS. Unfortunately, this had led to the appearance of a number of papers on, e.g. interferences in AAS which were essentially a repetition, through ignorance, of work that had been done previously in old "flame photometry." A similar situation may arise again since the inductively-coupled plasma has become a popular excitation source in atomic spectroscopy. For example, some of the current studies on sample nebulization do not seem to produce essentially new information.

[12] D. S. Gough and P. Hannaford, Spectrochim. Acta 35B, 677 (1980).

[13] P. Hannaford and R. G. Horn, J. Phys. C: Solid State Phys. 6, 2223 (1973).

[14] C. S. RanN, Spectrochim. Acta 23B, 827 (1968).

[15] H. L. Kahn and W. Slavin, Appl. Opt. 2, 931 (1963).

[16] C. Th. J. Alkemade, Appl. Opt. 7, 1261 (1968).

[17] A. Walsh, Atom. Absorption Newsletter 17, 97 (1978). 
(iii) Most physical methods of analysis have evolved from experimental methods that were originally in use in fundamental or applied physics or physical chemistry. In addition to atomic absorption and emission spectrometry, we can mention, for instance, atomic fluorescence spectrometry, mass spectrometry, nuclear magnetic resonance and microwave spectroscopy. There is necessarily a time-lapse between the use of these methods in physics etc. and their introduction in analytical chemistry (for a more detailed discussion see Ref. [18]). This applies also to the analytical application of special instrumental components or measuring "tricks" in atomic spectroscopy; we mention, e.g. the use of a furnace or sputtering chamber as atomizer or the technique of wavelength-modulation. In recent years this time-lapse has tended to become significantly shorter. But it would still be worthwhile to scan more systematically the experimental techniques currently in use in atomic or molecular physics, combustion research, astrophysics, etc., to see whether they might not be exploited in analytical chemistry. As a result of such a scan a suggestion was once made [18] to transfer ion mass spectrometry as applied in flame research with a quadrupole mass spectrometer [19] to chemical analysis. (In fact, GRAY [20] had developed this method, independently, for the analysis of solutions nebulized into a small d.c. arc.) We should follow more closely the discovery of new effects and the development of new instruments in physical research, which could be profitable in chemical analysis.

(iv) Once a physical method has been taken over by chemical analysts, the continuous striving by commercial firms or individual researchers to improve this method with regard to sensitivity, stability and ease of operation, in turn, profits fundamental research. The improvements of analytical atomic spectroscopy, in particular the perfecting of hollow-cathode lamps and sputtering chambers, have already yielded profitable returns, e.g. in the study of excitation and quenching processes in metal vapours. AAS and AFS have now become a familiar tool for measuring the population of atomic ground- and excited levels, under stationary conditions as well as a function of time (for examples see [21]). In our laboratory the technique of AAS with a boosted, pulsed hollow-cathode lamp and a xenon-arc lamp has recently been successfully applied by H. VAN DIJK to measure accurately curves-of-growth for $\mathrm{Cr}$ absorption lines in a shock tube. We also recall that, conversely, dye lasers were first tried as excitation sources in analytical fiame fluorescence spectroscopy [22] before they were used in fundamental studics on metal vapours in flames.

(v) The historical development of AAS since 1955 shows that the mere proposal and demonstration of a new method is not sufficient to effect its actual application in analysis. In the beginning analytical spectroscopists were rather slow in grasping the universal potentialities of a method that has now become the most widely applied one in analytical spectroscopy. The birth of a ncw idea alone does not automatically lead to its acceptance as a member of an established family. An adequate follow-up, including the propagation of the idea, the optimization of the necessary equipment and its trying-out in practice are at least as important as the birth. In this respect the efforts that have been made at the CSIRO, for example, to construct simple, practical hollow-cathode lamps for various elements have been a decisive factor in the success of AAS.

\section{CONCLUSION}

Especially in regard to the last point (v), raised above, nobody will hesitate to acknowledge Sir Alan WaLSH as the "Father of AAS." My real (but late) "discovery"

[18] C. Th. J. Alkemade, Proc. Soc. Anal. Chem. 10, 130 (1973).

[19] P. F. KnewstubB, Mass Spectrometry of Organic Ions, Chapter 6, p. 255. Academic Press, New York (1963).

[20] A. L. Gray, The Analyst 100, 289 (1975); Anal. Chem. 47, 600 (1975).

[21] A. FontIJN and W. FELdER, Reactive Intermediates in the Gas Phase. Generation and Monitoring (edited by D. W. SetSer), Chapter 2, p. 59. Academic Press, New York (1979).

[22] L. M. Frazer and J. D. Winefordner, Anal. Chem. 43, 1693 (1971). 
in this field was mainly my realization that the absorption flame photometer described in our 1955 paper was an important invention-important, but thanks to the pioneering work of WALSH and his associates.

Acknowledgement-The author acknowledges the very useful lessons he has had from SHEILA MCNAB in the improvement of the English style and idiom of this prose. 